\title{
Stabilizing Schemes for Piecewise-Linear Reduced Order Models via Projection and Weighting Functions
}

\author{
Bradley N Bond \\ Research Laboratory in Electronics, \\ Massachusetts Institute of Technology, \\ 77 Massachusetts Ave, Cambridge, MA, 02139 \\ Email: bnbond@mit.edu
}

\author{
Luca Daniel \\ Research Laboratory in Electronics, \\ Massachusetts Institute of Technology, \\ 77 Massachusetts Ave, Cambridge, MA, 02139 \\ Email: luca@mit.edu
}

\begin{abstract}
In this paper we present several results concerning the stabilization of piecewise-linear reduced order models. We include proofs of internal and external stability for models whose system matrices possess special structures. We then introduce a new projection scheme, and a new set of weighting functions which allow us to extend some of these results to piecewiselinear systems comprised of arbitrary matrices, at least one of which is Hurwitz. Included are an algorithm for creating switching piecewise-linear reduced models comprised of globally exponentially stable systems, and stable simulation results for a system which produces unstable results when using the standard TPWL method.
\end{abstract}

\section{INTRODUCTION}

Recently there has been a large amount of interest in piecewise-linear (PWL) model order reduction (MOR) techniques [1], [2], [3], [4], [5], [6], [7], [8]. Currently, PWL methods, such as the Trajectory Piecewise-Linear (TPWL) method [9] are probably the only viable known way to handle strongly nonlinear systems. The issue of stability in TPWL models has been studied [10], however, no algorithms are yet available that guarantee the stability of such reduced order models (ROMs).

The stability of dynamical systems was studied extensively by Lyapunov over one hundred years ago. Lyapunov's work showed that a system's equilibrium point is stable if there exists a function, termed 'Lyapunov function', which satisfies certain conditions along the system trajectories [11]. For linear systems described by Hurwitz matrices, it has been shown [12] that there always exists a quadratic Lyapunov function. Recent results provide conditions for the existence of Lyapunov functions for hybrid switching systems [13], however such conditions implicitly impose strong constraints on the structure of the individual linear models.

The construction of stable ROMs for linear systems has also been a problem of great interest [14], [15], [16], [17]. For projection-based methods, one can always find an orthogonal projection which preserves system stability. This result is based on the fact that a quadratic Lyapunov function for the original system implies the existence of a quadratic Lyapunov function for the reduced system. Methods have also been proposed which use optimization-based fitting techniques to preserve system stability [18], [19].

In this work we first present a proof of stability for PWL models comprised of linearized systems which are all described by matrices with some special structure. We then present a projection scheme which preserves stability for any PWL system that permits a quadratic Lyapunov function (Section III). Applications possessing this property include for instance nonlinear circuits assembled entirely using two-port passive devices with monotonic I-V relationships (e.g. diodes, nonlinear capacitors, nonlinear resistors), or systems including arbitrary smooth nonlinearities which are not too strong relative to the linear part. Such systems produce linearized models described by diagonally-dominant Hurwitz matrices. In addition, we present a new projection scheme, and a set of weighting functions which allow us to extend some of our results to PWL models comprised of arbitrary matrices (Section IV). The remainder of the paper presents an algorithm for creating such stabilized models (Section V), and numerical results supporting our algorithm (Section VI).

\section{BACKGROUND}

\section{A. System Stability}

In this section we summarize definitions and some basic results about different types of system stability and their relationships. Consider the nonlinear dynamical system

$$
\dot{x}=f(x, u), \quad y=g(x)
$$

with equilibrium point $x_{e q}=0$ such that

$$
f(0,0)=0, \quad \forall t .
$$

Here $x \in \mathbb{R}^{N}$ is the state vector, and $u \in \mathbb{R}, y \in \mathbb{R}$ are the input and output respectively. The internal stability of System (1) is defined by the attractiveness of the equilibrium point of the autonomous system

$$
\dot{x}=f(x, 0) .
$$

Specifically, the equilibrium point is said to be exponentially stable if all solutions starting from arbitrary initial conditions 
converge to the equilibrium point exponentially fast. Such internal stability property can be shown by the existence of a Lyapunov function - a non-negative function which decreases monotonically along all system trajectories.

Theorem 2.1 ([11]): The equilibrium point $x_{e q}=0$ of system (1) is globally exponentially stable if there exist constants $\lambda_{1}, \lambda_{2}, \lambda_{3}>0$ and a continuously differentiable Lyapunov function $L(x, t)$ such that

$$
\begin{aligned}
\lambda_{1} x^{T} x \leq L(x, t) & \leq \lambda_{2} x^{T} x \\
\frac{\partial}{\partial t} L(x, t) & \leq-\lambda_{3} x^{T} x
\end{aligned}
$$

$\forall t \geq 0, \forall x \in \mathbb{R}^{n}$.

We consider $x_{e q}=0$ without loss of generality because the coordinate system can always be redefined in order to translate the equilibrium point to the origin of a new coordinate system.

For a linear system

$$
\dot{x}=A x+b u(t)
$$

with Hurwitz matrix $A$, defined as a matrix whose eigenvalues all have negative real part, i.e. $\operatorname{Re}(\lambda(A))<0$, there always exists a quadratic Lyapunov function $L(x)=x^{T} P x$, where $P$ is a positive definite matrix which solves the Lyapunov equation

$$
P A+A^{T} P=-Q
$$

for some positive-definite matrix $Q \succ 0$.

System (1) is said to be externally stable if the system's output $y(t)$ can be bounded by a function of the system's input $u(t)$. Specifically, the system is said to be small signal input/output stable if there exist constants $r_{2}>0$ and $\gamma_{2}<\infty$ such that

$$
\|y\|_{2} \leq \gamma_{2}\|u\|_{2}
$$

for all $t>t_{0}$ given initial state $x(0)=0$ and input $u(t)$ such that $\|u\|_{\infty}<r_{2}$.

The external and internal stability of System (1) are connected by the following theorem.

Theorem 2.2 ([11]): Suppose $x=0$ is an exponentially stable equilibrium of system (1), $F(x, u)$ is continuously differentiable, and $F(x, u), g(x, u)$ are locally Lipschitz continuous at $(0,0)$, i.e. suppose there exist finite constants $k_{f}, k_{g}, c_{2}$ such that

$$
\begin{aligned}
\|F(x, u)-F(z, v)\| & \leq k_{f}[\|x-z\|+\|u-v\|] \\
\|g(x, u)-g(z, v)\| & \leq k_{g}[\|x-z\|+\|u-v\|]
\end{aligned}
$$

$\forall(x, u),(z, v) \in B_{c_{2}}(0)$ and $\forall t \geq 0$, then system (1) is smallsignal input/output stable. Here, $B_{c_{2}}(0)$ is a ball of radius $c_{2}$ centered at the origin.

\section{B. Stable Model Order Reduction for Linear Systems}

For stable linear systems, there are several projection methods which are known to preserve stability. Here we consider one of such techniques.

Theorem 2.3 ([20]): Consider a linear system

$$
\dot{x}=A x+b u
$$

where $A$ is a Hurwitz matrix. Let $P$ and $Q$ be symmetric positive-definite matrices which solve (5), and let $V$ be an orthonormal projection matrix such that $x=V \hat{x}$ and $x \in \mathbb{R}^{q}$ where $q<<N$. If $U$ is defined by

$$
U^{T}=\left(V^{T} P V\right)^{-1} V^{T} P,
$$

and $\hat{A}=U^{T} A V$, then the reduced order system

$$
\dot{\hat{x}}=\hat{A} \hat{x}+\hat{b} u
$$

is stable.

Proof. To prove the stability of (11), it is sufficient to show that

$$
\hat{P} \hat{A}+\hat{A}^{T} \hat{P}=-\hat{Q}
$$

for some symmetric positive-definite $\hat{P}$, and symmetric positive-definite $Q$. Let's select symmetric and positivedefinite matrix $\hat{P}=V^{T} P V$. By the definition of $U$,

$$
\hat{P} \hat{A}=V^{T} P V U^{T} A V=V^{T} P A V,
$$

and thus

$$
\hat{P} \hat{A}+\hat{A}^{T} \hat{P}=V^{T}\left(P A+A^{T} P\right) V=-V^{T} Q V \prec 0 .
$$

Thus (12) is satisfied for symmetric positive-definite matrix $\hat{Q}=V^{T} Q V \square$.

\section{TPWL Model Order Reduction for Nonlinear Systems}

Consider a nonlinear system of order $N$

$$
\dot{x}=f(x)+b u, \quad y=c^{T} x,
$$

whose vector field $f(x)$ can be approximated in some important regions of the state-space by a convex combination of affine functions

$$
f(x)=\sum_{i} w_{i}(x)\left[A_{i} x+K_{i}\right]
$$

where

$$
A_{i}=\left.\frac{\partial f(x)}{\partial x}\right|_{x_{i}} \quad K_{i}=f\left(x_{i}\right)-A_{i} x_{i}
$$

are linearizations of $f(x)$, and $w_{i}(x)$ are weighting functions such that $w_{i} \in[0,1]$ and $\sum_{i} w_{i}=1$. It is possible to introduce a linear projection $x=V \hat{x}$, where $\hat{x} \in \mathbb{R}^{q}$ and $q<<N$, such that each linear system is projected into a subspace creating the piecewise-linear reduced order model

$$
\dot{\hat{x}}=\sum_{i} w_{i}(\hat{x})\left[\hat{A}_{i} \hat{x}+\hat{K}_{i}\right]+\hat{b} u, \quad y=\hat{c}^{T} \hat{x}
$$

in which we have defined $\hat{A}_{i}=V^{T} A_{i} V, \hat{b}=V^{T} b$, and $\hat{c}=V^{T} c$.

In standard trajectory piecewise-linear methods [9], the linearization points $x_{i}$ are chosen as states along trajectories which solve system (1) when driven by some typical inputs $u(t)$. There are many possible options for generating the columns of the projection matrix $V$. For instance, one can use Krylov vectors of the individual linearized systems [9], [1], important states from the trajectories [21], or TBR vectors [2].

It is important to notice here that the procedure above may potentially produce unstable reduced models from originally 
stable systems. Such instabilities may arise in three places: Jacobian matrices $A_{i}$ of stable nonlinear systems are not guaranteed to be Hurwitz; $V^{T} A_{i} V$ is not guaranteed to be Hurwitz even if $A_{i}$ is Hurwitz; finally, positive sums of Hurwitz matrices $\sum_{i} w_{i} A_{i}$ are not guaranteed to be Hurwitz.

\section{Preservation of Stability in PWL Models}

It is difficult to prove internal stability for reduced order PWL models due to the potential instabilities introduced by the linearizations, summation of matrices, and projection. However, there exist many cases where large-order systems comprised of matrices possessing some special structure can be shown to be stable. In such cases there may also exist projection schemes which create stable ROMs. In this section we shall identify some classes of stable PWL models and then describe a projection scheme which preserves stability.

To begin, we consider the PWL model

$$
\dot{x}=\sum_{i} w_{i}(x)\left[A_{i} x+K_{i}\right]+b u \quad y=g(x),
$$

and note that it can be rewritten as

$$
\dot{x}=\sum_{i} w_{i}(x) A_{i} x+B(x) u \quad y=g(x),
$$

where

$$
B(x) u=\left[\begin{array}{ll}
b & \sum_{k} w_{i}(x) K_{i}
\end{array}\right]\left[\begin{array}{l}
u_{1} \\
u_{2}
\end{array}\right]
$$

is a state-dependent input matrix. System (19) is obtained by selecting $u_{2}=1 \forall t$. Thus systems of the form (19) are a subset of systems of the form (20), and any stability results which apply to the latter also apply to the former.

\section{A. Structured System Matrices}

Often times the system matrices $A_{i}$ will all share some nice structure due to the fact that they are all linearizations of the same nonlinear function. In such cases it may be possible to find a Lyapunov function which proves internal stability of the PWL system. Consider a PWL system comprised of symmetric Hurwitz matrices. Such systems are encountered for instance when using TPWL reduction on nonlinear circuits comprised of nonlinear elements such as nonlinear resistors, nonlinear capacitors, and diodes.

Prop. 3.1: If each $A_{i}$ in System (20) is symmetric and Hurwitz, and $w_{i}(\hat{x}): \mathbb{R}^{q} \mapsto[0,1]$ are continuously differentiable functions such that $\sum_{i} w_{i}=1$, then System (20) has a globally exponentially stable equilibrium point at the origin.

Proof. Consider $L(x)=x^{T} x$ as a candidate Lyapunov function for the system. Clearly $L(x)$ satisfies condition (3), so we simply need to verify that $\frac{\partial L}{\partial t} \leq-\lambda_{3} x^{T} x$. Since $A_{i}$ are all symmetric negative-definite,

$$
\frac{\partial L(x)}{\partial t}=2 \sum_{i} w_{i}(x) x^{T} A_{i} x \leq-2 \min _{i} \sigma_{\min }\left(A_{i}\right) x^{T} x
$$

where $\sigma_{\min }\left(A_{i}\right)$ is the smallest singular value of $A_{i}$. Thus, condition (4) is satisfied by selecting $\lambda_{3}=2 \min _{i} \sigma_{\min }\left(A_{i}\right)$, hence $L(x)$ is a Lypaunov function for System (20) $\square$.
We next consider system matrices $A_{i}$ which are Hurwitz and have a structure such that $A_{i}+A_{i}^{T}$ is symmetric and Hurwitz. For example, Hurwitz matrices which are diagonallydominant but not necessarily symmetric. Such matrices could result from, for instance, the nodal analysis of analog circuits possessing a mixture of linear elements (some of which connect all nodes to ground), which generate a Hurwitz diagonally-dominant symmetric Jacobian, and also transistors, which generate non-symmetric elements in the Jacobian.

Prop. 3.2: If each matrix $A_{i}$ in System (20) is such that $A_{i}+A_{i}^{T}$ is Hurwitz and symmetric, and $w_{i}(\hat{x}): \mathbb{R}^{q} \mapsto[0,1]$ are continuously differentiable functions such that $\sum_{i} w_{i}=1$, then System (20) has a globally exponentially stable equilibrium point at the origin.

Proof. consider the candidate Lyapunov function $L(x)=$ $x^{T} x$.

$$
\frac{\partial L(x)}{\partial t}=2 \sum_{i} w_{i}(x) x^{T} A_{i} x=\sum_{i} w_{i}(x) x^{T}\left(A_{i}+A_{i}^{T}\right) x
$$

By definition of $A_{i}, A_{i}+A_{i}^{T}$ is symmetric negative-definite, so the remainder of the proof follows exactly as in the previous section $\square$. Thus, for systems comprised of structured Hurwitz matrices, it may be possible to find a quadratic Lyapunov function which proves internal stability.

\section{B. Stability of Reduced Order Models}

We will now show that it is possible to reduce systems described in the previous section to obtain a guaranteed stable ROM. In fact, there exists a projection which guarantees stability of the reduced model created from any stable system which permits a quadratic Lyapunov function.

Prop. 3.3: If there exists a quadratic Lyapunov function $L(x)=x^{T} P x$ which satisfies conditions (3) and (4) for system (20), then given any right-projection matrix $V$, there exists a left-projection matrix $U$ such that the ROM

$$
\dot{\hat{x}}=\sum_{i} w_{i}(\hat{x}) \hat{A}_{i} x+\hat{B}(\hat{x}) u \quad y=\hat{g}(\hat{x}),
$$

where $\hat{A}_{i}=U^{T} A_{i} V$, has a globally stable equilibrium point at the origin.

Proof. Consider $U$ as described in Section II-B

$$
U^{T}=\left(V^{T} P V\right)^{-1} V^{T} P,
$$

which results in $U^{T} V=I$. Now consider $\hat{L}(\hat{x})=\hat{x}^{T} V^{T} P V \hat{x}$ as a Lyapunov function for system (22).

$$
\frac{\partial \hat{L}(\hat{x})}{\partial t}=\sum_{i} w_{i}(\hat{x}) \hat{x}^{T} V^{T} P V U^{T} A_{i} V \hat{x} .
$$

Substituting (23) into (24) yields

$$
\frac{\partial \hat{L}(\hat{x})}{\partial t}=\sum_{i} w_{i}(\hat{x}) \hat{x}^{T} V^{T} P A_{i} V \hat{x}=\frac{\partial L(V \hat{x})}{\partial t}
$$

which was assumed to satisfy (4), so the reduced system is stable $\square$. For structured systems described in the previous section, we find that $U=V$. 


\section{Input-Output Stability}

In the previous two sections we showed several cases where the autonomous systems are exponentially stable, and that this property can be preserved in the ROM. Exponential stability is desirable because input/output stability follows from Theorem 2.2.

Prop. 3.4: Consider System (20) where $L(x)=x^{T} P x$ is a Lyapunov function which satisfies (3) and (4), $\hat{A}_{i}=U^{T} A_{i} V$, $\hat{b}=U^{T} b, \hat{K}_{i}=U^{T} K_{i}$ for some orthonormal projection matrix $V \in \mathbb{R}^{N \times q}, U$ as defined in (30), and $\hat{g}(\hat{x})$ is Lipschitz continuous and smooth. If $w_{i}(\hat{x}): \mathbb{R}^{q} \mapsto[0,1]$ are continuously differentiable functions such that $\sum_{i} w_{i}=1$, then System (22) is small signal input/output stable.

Proof. We shall prove this proposition by applying Theorem 2.2 and using the fact that the autonomous system is exponentially stable, as shown in Section III-B. Thus, we merely need to show that

$$
\begin{aligned}
F(\hat{x}, u) & =\sum_{i} w_{i}(\hat{x}) \hat{A}_{i}(\hat{x})+\hat{\tilde{B}}(\hat{x}) u \\
g(\hat{x}, u) & =\hat{c}^{T} \hat{x}
\end{aligned}
$$

are continuously differentiable and locally Lipschitz continuous at $(0,0)$, as defined in (7).

The output function $g(\hat{x}, u)$ is defined to be Lipschitz in $\hat{x}$, so it is continuously differentiable and Lipschitz with constant $k_{g}$. Similarly, the vector field $F(\hat{x}, u)$ is continuously differentiable because it is a sum of products of continuously differentiable functions.

It can be shown that Lipschitzness of $F(\hat{x}, u)$ is satisfied by selecting

$$
k_{f}=\sum_{i}\left[L_{w}\left\|\hat{A}_{i}\right\|+\left(c_{2} L_{w}+1\right)\left\|\hat{K}_{i}\right\|\right]+\|\hat{b}\|,
$$

where $c_{2}$ is a bound on the input and $L_{w}$ is the Lipshcitz constant for the weighting functions. Thus, Theorem (2.2) is applicable, and system (22) is small signal input/output stable $\square$. It follows that the system

$$
\dot{\hat{x}}=\sum_{i} w_{i}(\hat{x})\left[\hat{A}_{i} \hat{x}+\hat{K}_{i}\right]+\hat{b} u \quad y=\hat{g}(\hat{x}),
$$

is also input/output stable.

\section{Weights AND PROJeCtion FOR THE SAKE OF STABILITY}

In the previous section it was shown that stable reduced order models can be created from system (20) when the system permits a quadratic Lyapunov function. However, it is possible that not all of the matrices will have a nice structure, and some of them many not even be Hurwitz. In this case, the associated system (20) may be unstable, even though it models a stable nonlinear system. Thus, it is desirable to "stabilize" the ROM by selecting an appropriate projection scheme, and set of weighting functions.

\section{A. Derivation of a New Piecewise-Linear Model}

Given a large-order piecewise-linear system, (20), we may introduce a linear projection $x=V \hat{x}$ such that

$$
V \dot{\hat{x}}=\sum_{i} w_{i}(\hat{x})\left[A_{i} V \hat{x}\right]+B(\hat{x}) u .
$$

To reduce the number of equations, we define a weighted piecewise-constant left-projection function

$$
U(\hat{x})=\sum_{k=1}^{\kappa} \mu_{k}(\hat{x}) U_{k}
$$

where $u_{k}(\hat{x}) \in[0,1], \sum_{k} u_{k}(\hat{x})=1$, and $U_{k} \in \mathbb{R}^{N \times q}$. Leftmultiplying (26) by $U(\hat{x})^{T}$ yields

$$
\begin{array}{r}
\sum_{k} \mu_{k}(\hat{x}) U_{k}^{T} V \dot{\hat{x}}=\sum_{k} \mu_{k}(\hat{x}) U_{k}^{T}\left(\sum_{i} w_{i}(\hat{x})\left[A_{i} V \hat{x}\right]+B(\hat{x}) u\right) \\
=\sum_{k} \sum_{i} \mu_{k}(\hat{x}) w_{i}(\hat{x}) U_{k}^{T} A_{i} V \hat{x}+\sum_{k} \mu_{k}(\hat{x}) U_{k}^{T} B_{k}(\hat{x}) u
\end{array}
$$

To simplify the notation, we define

$$
\begin{aligned}
\hat{E}_{k} & =U_{k}^{T} V & \hat{B}_{k}(\hat{x}) & =\mu_{k}(\hat{x}) U_{k}^{T} B(x) \\
\hat{A}_{k i} & =U_{k}^{T} A_{i} V & & \hat{\tilde{B}}(\hat{x})=\sum_{k} \hat{B}_{k}(\hat{x}) \\
\hat{c} & =V^{T} c & &
\end{aligned}
$$

resulting in the final reduced system

$$
\sum_{k} u_{k}(\hat{x}) \hat{E}_{k} \dot{\hat{x}}=\sum_{k} \sum_{i}\left[\mu_{k}(\hat{x}) w_{i}(\hat{x}) \hat{A}_{k i}\right] \hat{x}+\hat{\tilde{B}}(\hat{x}) u
$$

\section{B. Weighting Functions for Stability}

For a system comprised of arbitrary matrices $A_{i}$ which are not all Hurwitz, the weighting functions must be specially constructed to ensure stability. Each Hurwitz matrix can be thought of as a stable system which may be somewhat perturbed by other nearby systems without losing stability. To this end, we may think of the PWL model as a collection of stable nonlinear systems centered around the different regions of the space. This results in a set of $M$ nonlinear vector fields which satisfy

$$
\dot{\hat{x}}=f_{m}(\hat{x})=\sum_{i} \sum_{k} \mu_{k}(\hat{x}) w_{i}^{m}(\hat{x}) \hat{A}_{k i} \hat{x}, \quad \text { for } x \in \mathbb{S}_{m},
$$

where $\mathbb{S}_{m}$ is a partition of the space containing the linearization point from which the Hurwitz matrix $A_{m}$ was created. We can then ensure that each of these nonlinear systems is exponentially stable, and select the weighting functions to create a single nonlinear system which switches between the stable subsystems.

Prop. 4.1: Consider system (20). If $V \in \mathbb{R}^{N \times q}$ is an orthonormal projection matrix, and at least one $A_{m}$ is Hurwitz, then there exist left-projection matrices $U_{k}$, piecewise-smooth functions $u_{k}(\hat{x}): \mathbb{R}^{q} \mapsto[0,1]$, piecewise-smooth switching functions $w_{i}(\hat{x}): \mathbb{R}^{q} \mapsto[0,1]$, and a partition of the state-space into $M$ polyhedra $\mathbb{S}_{m}$ where $M$ is the number of Hurwitz large matrices $A_{i}$, such that system (22) is comprised of $M$ 
exponentially stable systems, and $\hat{A}_{k i}=U_{k}^{T} A_{i} V, \hat{b}_{k}=U_{k}^{T} b$, $\hat{K}_{k i}=U_{k}^{T} K_{i}$, and $\hat{c}=V^{T} c$.

To begin, we partition the state-space into $M$ regions centered around the linearization points $x_{i}$ corresponding to the $M$ Hurwitz Jacobian matrices $A_{i}$. The partitions are defined as the polyhedra $\mathbb{S}_{m}$ such that

$$
\mathbb{S}_{m}=\left\{\hat{x} \in \mathbb{R}^{q} \mid \hat{x}_{m}=\operatorname{argmin}\left\{\min _{\hat{x}_{i}}\left(\| \hat{x}-\hat{x}_{i}||\right)\right\}\right\} .
$$

These partition polyhedra may be unbounded and the union of all partitions covers the entire state-space. In addition, we define $\Delta_{m}$ as half the maximum distance between linearization point $\hat{x}_{m}$ and all other linearization points $\hat{x}_{i}$

$$
\Delta_{m}=\frac{1}{2} \max _{i}\left\{\left\|\hat{x}_{m}-\hat{x}_{i}\right\|\right\} .
$$

If we define, as stated in Section II-B, the matrices

$$
U_{m}^{T}= \begin{cases}\left(V^{T} P_{m} V\right)^{-1} V^{T} P_{m} & \text { if } m \in \mathbb{I} \\ V^{T} & \text { if } m \notin \mathbb{I}\end{cases}
$$

where

$$
\mathbb{I}=\left\{m \in\{1, \ldots, \kappa\} \mid A_{m} \text { is Hurwitz }\right\},
$$

and $P_{m}$ solve (5) for $A_{m}$, then $\hat{E}_{m}=U_{m}^{T} V=I$ for all $m$, and $\hat{A}_{m m}$ is Hurwitz. This simplifies the reduced model to

$$
\dot{\hat{x}}=\sum_{k} \sum_{i}\left[\mu_{k}(\hat{x}) w_{i}(\hat{x}) \hat{A}_{k i}\right] \hat{x}+\hat{\tilde{B}}(\hat{x}) u
$$

and each partition corresponds to a stable matrix.

By bounding the weighting function such that each stable matrix is not perturbed unstable by the surrounding systems, we can guarantee exponential stability of each partition model. It can be shown that each autonomous partition system $\dot{\hat{x}}=$ $f_{m}(\hat{x})$ permits the Lyapunov function $L_{m}(x)=x^{T} P_{m} x$, and thus has a globally exponentially stable equilibrium point at the origin, provided we enforce the constraint that $w_{m}^{m} \geq w_{m}^{*}$ for all $x$, where

$$
w_{m}^{*}=\frac{\sum_{k} \sum_{i \neq m} \mu_{k}(\hat{x}) \sigma_{\max }\left(\hat{P}_{m} \hat{A}_{k i}\right)}{\mu_{m}(\hat{x}) \sigma_{\min }\left(\hat{P}_{m} \hat{A}_{m m}\right)+\sum_{k} \sum_{i \neq m} \mu_{k}(\hat{x}) \sigma_{\max }\left(\hat{P}_{m} \hat{A}_{k i}\right)} .
$$

The final model will switch between vector fields $f_{m}(\hat{x})$ based on which partition the state is in, therefore we define a single set of weighting functions $w_{i}(\hat{x})$ which satisfy $w_{m}(\hat{x}-$ $\left.\delta_{m}\right)=w_{m}^{*}$ for all $\delta_{m}$ such that $\left\|\delta_{m}\right\|=\left\|\Delta_{m}\right\|$ when $\hat{x} \in \mathbb{S}_{m}$. Additionally, we require that $w_{m}^{m}\left(x_{m}\right)=1$, and that the weights decay exponentially from the dominant linearization point $x_{m}$. One possible set of functions which satisfy such constraints are

$$
w_{i}(x)=\frac{\exp \left\{\beta_{m}(\hat{x})\left(\hat{x}-\hat{x}_{i}\right)^{T}\left(\hat{x}-\hat{x}_{i}\right)\right\}}{\sum_{j} \exp \left\{\beta_{m}(\hat{x})\left(\hat{x}-\hat{x}_{j}\right)^{T}\left(\hat{x}-\hat{x}_{j}\right)\right\}}
$$

where

$$
\beta(\hat{x})=\left\{\frac{\log \left(w_{m}^{*}\right)}{\Delta_{m}^{2}} \mid m=\underset{i \in \mathbb{I}}{\operatorname{argmin}}\left\{\left\|\hat{x}-\hat{x}_{i}\right\|\right\}\right\}
$$

switches discretely across the partition borders.
The constraint on $\mathbb{I}$ allows us to consider non-Hurwitz reduced matrices $\hat{A}_{u}$ as perturbations to the dominant linear model, e.g. we consider $w_{q} A_{q}+w_{u} A_{u}$ where $A_{q}$ is the dominant model with $w_{q}>\frac{1}{2}$ and $A_{u}$ is non-Hurwitz, but we do not allow the non-Hurwitz matrix to be a dominant linear model, e.g. $w_{u}(x)<\frac{1}{2} \forall x$. This ensures that the system still satisfies the Lyapunov constraints without completely disregarding the unstable interpolation model, which would sacrifice accuracy in the approximation of the original vector field $F(x)$.

To completely specify the reduced order system (28), we must specify a set of left-projection weights $\mu_{k}(\hat{x})$. One possible choice of $\mu_{k}(\hat{x})$ which simplifies the weighting functions $w_{i}(\hat{x})$ is

$$
\mu_{q}(\hat{x})= \begin{cases}1 & \text { if } \hat{x} \in \mathbb{S}_{q} \\ 0 & \text { if } \hat{x} \notin \mathbb{S}_{q},\end{cases}
$$

which gives the weighting function bound

$$
w_{m}^{*}=\frac{\sum_{i \neq m} \sigma_{\max }\left(P_{m} A_{m i}\right)}{\sigma_{\min }\left(P_{m} A_{m m}\right)+\sum_{i \neq m} \sigma_{\max }\left(P_{m} A_{m i}\right)} .
$$

This choice of $u_{k}$ greatly reduces the dominant weight bound $w_{m}^{*}$ by eliminating all of the cross terms $\hat{A}_{k i}$ for $k \neq i$, thus allowing smoother transitions between the regions.

Thus, by selecting $U_{k}$ as defined in (30) and $w_{i}(\hat{x})$ as defined in (34), each autonomous system (29) has a globally exponentially stable equilibrium point at the origin. Guaranteeing stability of the switching model for arbitrary switching sequences will require additional constraints on the switching between partitions, which we are not considering in this work. However, it is unlikely in practice that switching will make the set of exponentially stable models unstable.

\section{STPWL ALGORITHM}

Using the above projection technique and weighting functions, we have developed an algorithm which produces PWL reduced models comprised of stable switching systems, created from an originally stable large-order nonlinear system. Our procedure, presented in Algorithm 1, is described as follows.

Given a stable nonlinear system

$$
\dot{x}=f(x)+b u,
$$

a training procedure is used to obtain $\kappa$ linear models. We require at least one of such linear models to be described by a Hurwitz matrix. In practice, the majority of linearizations of typical stable systems will be Hurwitz matrices. In particular, a linearization around the equilibrium point $x=0$ of system (36) will always produces a Hurwitz matrix [10]. Information from the trajectories and linear models is then used to construct an orthonormal projection matrix $V$, using for instance one of the methods mentioned in Section (II-C). For each Hurwitz matrix $A_{m}$, a Lyapunov equation (5) is solved to obtain a positivedefinite matrix $P_{m}$, which is then used to compute the leftprojection matrix $U_{m}=\left(V^{T} P_{m} V\right)^{-1} V^{T} P_{m}$, as defined in (30). However, since the computation of $U_{m}$ requires only $V^{T} P_{m}$, as opposed to $P_{m}$, any part of $P_{m}$ which lies in the null space 


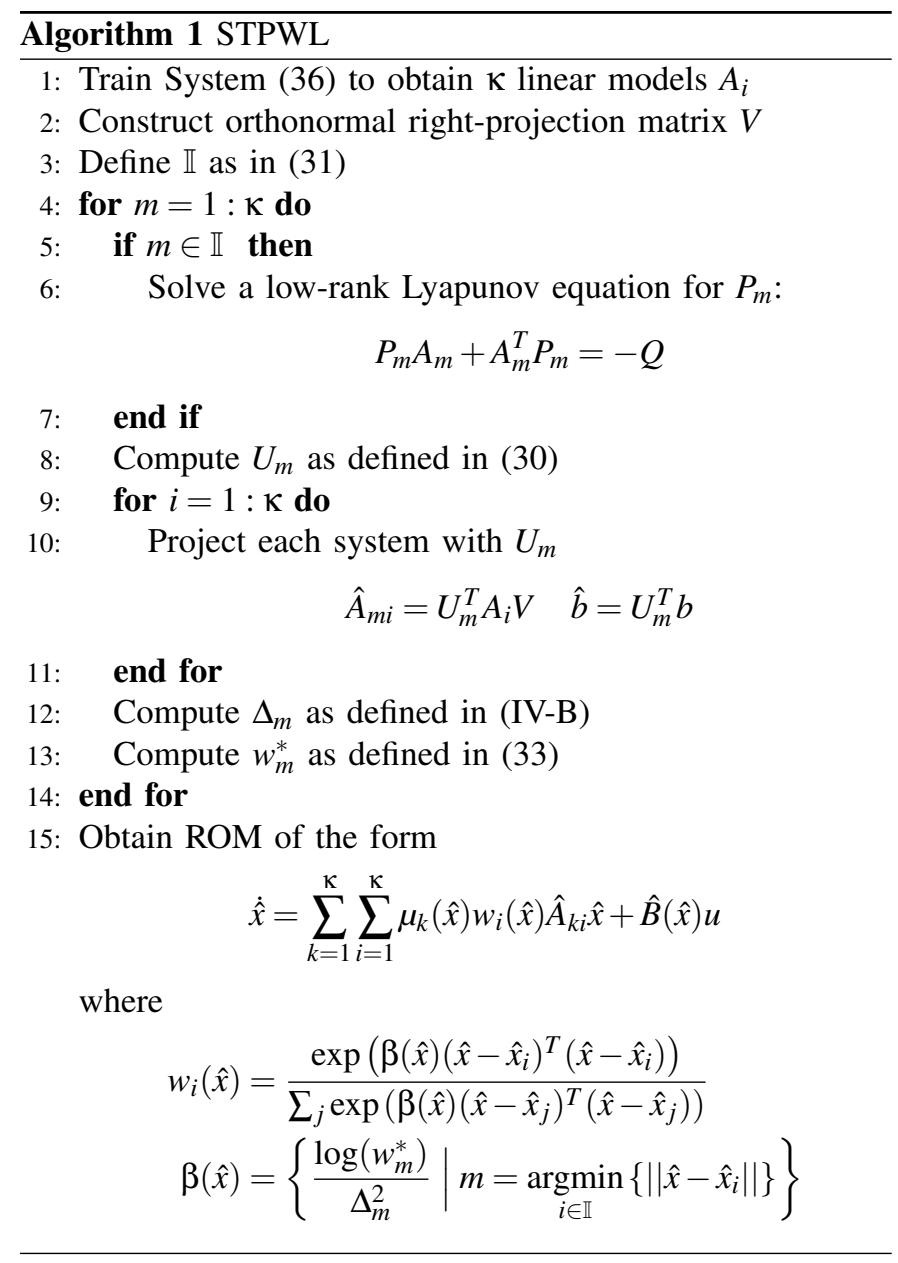

of $V^{T}$ will be eliminated. Thus, it is sufficient to solve a lowrank Lyapunov equation to obtain a rank- $q P_{m}$ with which the left-projection matrices can be computed. The resulting $\hat{P}_{m}=V^{T} P_{m} V$ and $\hat{A}_{m i}$ are used to compute weighting function bounds, as defined in (33), for each dominant linearization point. Lastly, a set of projection weights $u_{k}$ are chosen such that $\sum_{i} \mu_{k}=1$.

\section{RESULTS}

The proposed algorithm was tested on a stable physical system which generates non-symmetric, and possibly nonHurwitz, Jacobian matrices. Such example is a micromachined switch MEMS device, which is well documented in [1], [2]. The physical system is described by the pair of nonlinear partial differential equations

$$
\begin{aligned}
\hat{E} I_{0} h^{3} w \frac{\partial^{4} z}{\partial x^{4}}-S_{0} h w \frac{\partial^{2} z}{\partial x^{2}} & =F_{\text {elec }}+\int_{0}^{w}\left(P-P_{a}\right) d y-\rho_{0} h w \frac{\partial^{2} z}{\partial t^{2}} \\
\nabla \cdot\left((1+6 K) z^{3} P \nabla P\right) & =12 \mu \frac{\partial(P z)}{\partial t}
\end{aligned}
$$

which may be discretized into $m$ sections lengthwise and $n$ sections widthwise to obtain

$$
\begin{aligned}
\frac{\partial x_{1}}{\partial t} & =\frac{x_{2}}{3 x_{1}^{2}} \\
\frac{\partial x_{2}}{\partial t} & =\frac{2 x_{2}^{2}}{3 x_{1}^{3}}-\frac{3 \varepsilon_{0}}{2 \rho_{0} h} v^{2}+\frac{3 x_{1}^{2}}{\rho_{0} h w} \int_{0}^{w}\left(x_{3}-p_{a}\right) d y \\
& +\frac{3 x_{1}^{2}}{\rho_{0} h w}\left[S_{0} h w \frac{\partial^{2} x_{1}}{\partial x^{2}}-E^{3} h^{3} w \frac{\partial^{4} x_{1}}{\partial x^{4}}\right] \\
\frac{\partial x_{3}}{\partial t} & =-\frac{x_{2} x_{3}}{3 x_{1}^{3}}+\frac{1}{12 \mu x_{1}} \nabla\left(\left(1+6 \frac{\lambda}{x_{1}}\right) x_{1}^{3} x_{3} \nabla x_{3}\right),
\end{aligned}
$$

where the state variables are chosen as $\mathbf{x}_{1}=z \in \mathbb{R}^{m}, \mathbf{x}_{2}=$ $\frac{\partial u^{3}}{\partial t} \in \mathbb{R}^{m}$, and $\mathbf{x}_{3}=P \in \mathbb{R}^{m n}$. A detailed description of these functions can be found in [10].

System (37) was trained with a series of sinusoidal inputs with frequencies near $30 \mathrm{MHz}$ to obtain a set of $\kappa$ linear models, and a right projection matrix $V$, which was constructed with a moment matching approach. From this point, two separate reduced models were created - one using the traditional TPWL projection technique with smooth weighting functions, referred to as the TPWL-ROM, and one using the procedure described in Algorithm 1, referred to as the STPWL-ROM.

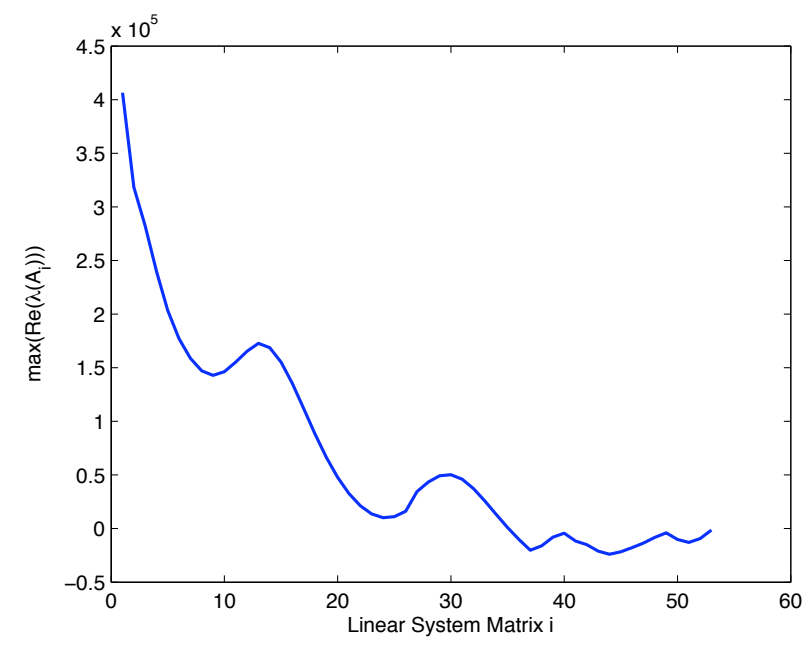

Fig. 1. Maximum real part of the eigenvalues of each system matrix $\hat{A}_{i}$ created from a Hurwitz matrix $A_{i}$ using the traditional TPWL method.

First we compare the Hurwitzness of the reduced linear model matrices created by the two reduction methods. Figure 1 plots the maximum real part of the eigenvalues of each reduced system matrix $\hat{A}_{m m}$ created from a Hurwitz matrix $A_{m}$ for the TPWL-ROM model. Despite each large matrix $A_{m}$ being Hurwitz, Figure 1 shows that in nearly every case the Hurwitzness was lost during projection. The eigenvalues for the STPWL-ROM however, as plotted in Figure 2, are all negative, resulting in Hurwitz reduced system matrices, as expected from Section IV-B.

The two reduced order models were then simulated with a set of sinusoidal inputs similar in frequency and amplitude 


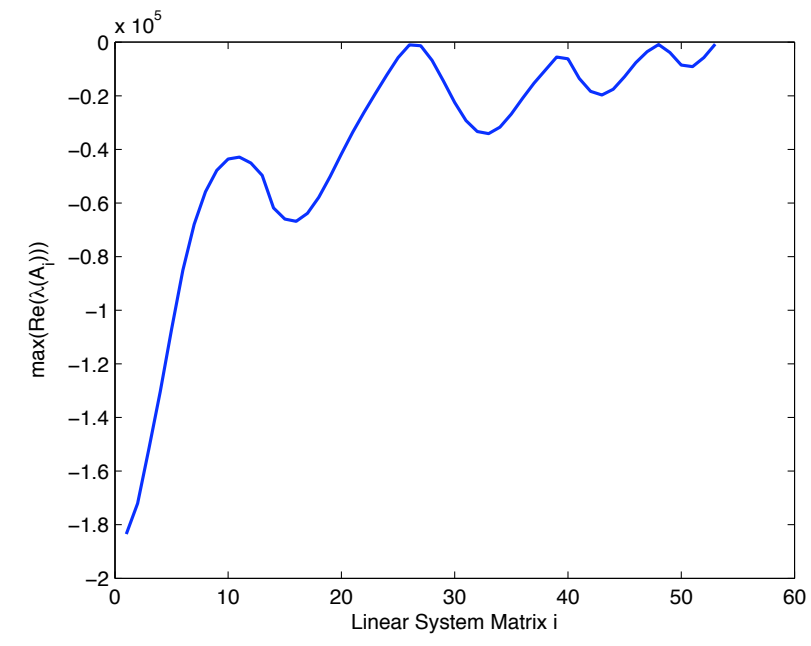

Fig. 2. Maximum real part of the eigenvalues of each system matrix $\hat{A}_{i}$ created from a Hurwitz matrix $A_{i}$ using the proposed projection method in Algorithm 1.

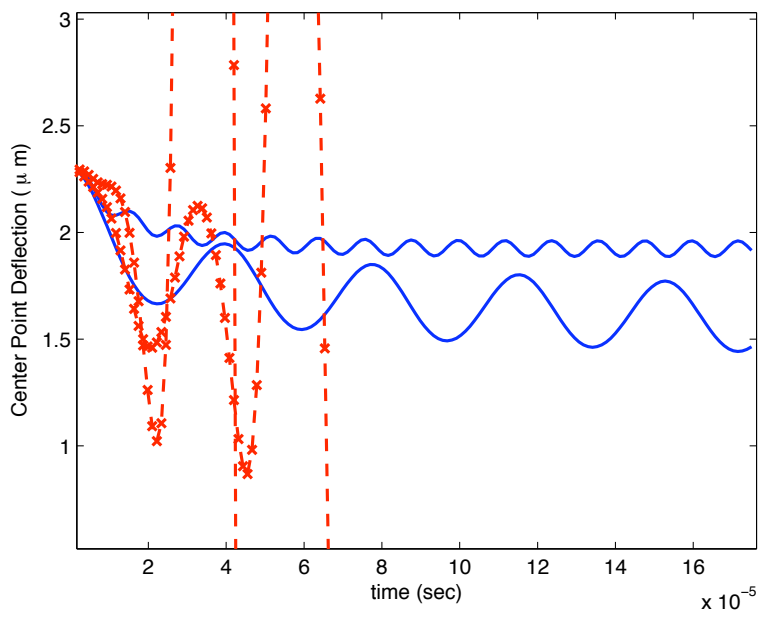

Fig. 3. Output of the TPWL-ROM driven by a set of inputs. The solid line indicates the output of the full nonlinear system, and the crosses indicate the ROM.

to the training inputs from which the linearized models were created. Figure 3 depicts the output of the TPWL-ROM for such inputs, and it is shown to explode. Hence, this model is unstable. However, Figure 4 plots the output of the STPWLROM for those same inputs, and it is seen that the STPWLROM produces stable outputs, which are also very accurate for those inputs.

\section{CONCLUSION}

In this paper we have presented proofs of internal and external stability for piecewise-linear reduced order models that are created from linear models described by system matrices with specific structures. Additionally, we have proposed a new projection scheme, and a new set of weighting functions which allow us to create, from arbitrary Hurwitz matrices, piecewiselinear reduced order models which are comprised of a set of guaranteed stable models. Lastly, we have presented results

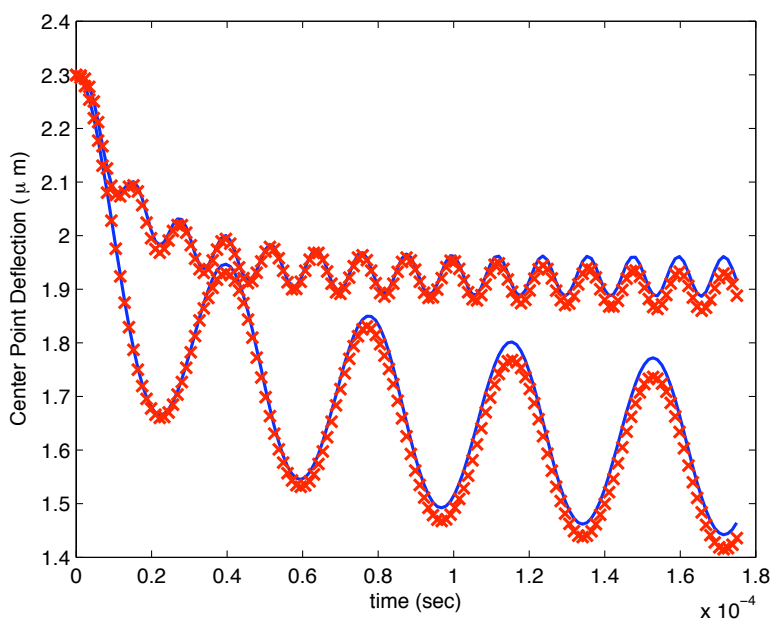

Fig. 4. Output of the STPWL-ROM driven by a set of inputs. The solid line indicates the output of the full nonlinear system, and the crosses indicate the ROM.

for one example where a stable nonlinear system produces unstable inaccurate reduced models using the traditional TPWL approach, while our proposed algorithm produces a reduced model with stable and accurate results.

\section{ACKNOWLEDGMENT}

This work was in part supported by MARCO, NSF, and DARPA.

\section{REFERENCES}

[1] M. Rewienski and J. White. A trajectory piecewise-linear approach to model order reduction and fast simulation of nonlinear circuits and micromachined devices. IEEE Trans. Computer-Aided Design, 22(2):155-70, Feb 2003.

[2] D. Vasilyev, M. Rewienski, and J. White. A tbr-based trajectory piecewise-linear algorithm for generating accurate low-order models for nonlinear analog circuits and mems. In Proc. of the ACM/IEEE Design Automation Conference, pages 490-5, June 2003.

[3] N. Dong and J. Roychowdhury. Piecewise polynomial nonlinear model reduction. In Proc. of the ACM/IEEE Design Automation Conference, June 2003.

[4] N. Dong and J. Roychowdhury. Automated extraction of broadly applicable nonlinear analog macromodels from spice-level descriptions. In Proc. of the IEEE Custom Integrated Circuits Conference, October 2004.

[5] S. Tiwary and R. Rutenbar. Scalable trajectory methods for on-demand analog macromodel extraction. In $42^{\text {nd }}$ ACM/IEEE Design Automation Conference, June 2005.

[6] N. Dong and J. Roychowdhury. Automated nonlinear macromodelling of output buffers for high-speed digital applications. In $42^{\text {nd }} A C M / I E E E$ Design Automation Conference, June 2005.

[7] B. Bond and L. Daniel. Parameterized model order reduction for nonlinear dynamical systems. In Proc. of the IEEE/ACM International Conference on Computer-Aided Design, pages 487-494, San Jose, CA, 2005.

[8] Y. Wan and J. Roychowdhury. Faster, parametric trajectory-based macromodels via localized linear reductions. In Proc. of IEEE/ACM International Conference on Computer Aided-Design, November 2006.

[9] M. Rewienski and J. K. White. A trajectory piecewise-linear approach to model order reduction and fast simulation of nonlinear circuits and micromachined devices. In Proc. of IEEE/ACM International Conference on Computer Aided-Design, pages 252-7, San Jose, CA, USA, November 2001 
[10] M. Rewienski. A trajectory piecewise-linear approach to model order reduction of nonlinear dynamical systems. P.hD. Thesis, Massachusetts Institute of Technology, June 2003.

[11] M. Vidyagasar. Nonlinear Systems Analysis. Prentice Hall, New York, 1978.

[12] Z. Gajic. The Lyapunov Matrix Equation in System Stability and Control. Academic Press, London, 1995.

[13] S. Pettersson and B. Lennartson. Exponential stability of hybrid systems using piecewise quadratic lyapunov functions resulting in lmis. Proc. of the 14th World Congress of IFAC, 11(11):103-108, Feb 1999.

[14] K. J. Kerns, I. L. Wemple, and A. T. Yang. Stable and efficient reduction of substrate model networks using congruence transforms. In Proc. of IEEE/ACM International Conference on Computer Aided-Design, pages 207 - 214, San Jose, CA, November 1995.

[15] L. M. Silveira, M. Kamon, I. Elfadel, and J. K. White. Coordinatetransformed arnoldi algorithm for generating guarantee stable reducedorder models of RLC. Computer Methods in Applied Mechanics and Engineering, 169(3-4):377-89, February 1999.

[16] A. Odabasioglu, M. Celik, and L. T. Pileggi. PRIMA: passive reducedorder interconnect macromodeling algorithm. IEEE Trans. ComputerAided Design, 17(8):645-654, August 1998.

[17] Zhaojun Bai, Peter Feldmann, and Roland W. Freund. Stable and passive reduced order models based on partial pade approximation via the lanczos process. Technical Report Numerical Analysis Manuscript No.97-3-10, Bell Laboratories, Lucent Technologies, Murray Hill, New Jersey, October 1997.

[18] C. Coelho, J. R. Phillips, and L. M. Silveira. On generating compact, passive models of frequency-described systems. In Symposium on Integrated Circuits and Systems, Porto Alegre, RS, Brazil, September 2002.

[19] K. C. Sou, A. Megretski, and L. Daniel. A quasi-convex optimization approach to parameterized model order reduction. In Proc. of the ACM/IEEE Design Automation Conference, CA, June 2005.

[20] A. Megretski. Lecture notes on projection-based model reduction. http://web.mit.edu/6.242/www/syll.html, September 2004.

[21] K. Willcox and J. Peraire. Balanced model reduction via the proper orthogonal decomposition. In Proceedings of the 15th AIAA Computational Fluid Dynamics Conference, Anaheim, CA, June 2001. 